\title{
Mental Fatigue Dimension
}

National Cancer Institute

\section{Source}

National Cancer Institute. Mental Fatigue Dimension. NCI Thesaurus. Code C132285.

A dimension of mental fatigue in the Multidimensional Fatigue Inventory. 